- This paper highlights to the practitioner her/his duty of care and the need for valid informed consent.

- Points to consider prior to, and when referring patients for extractions under general anaesthesia.

- This paper also highlights to the practitioner the need to keep contemporaneous notes.

\title{
Appropriate consent and referral for general anaesthesia - a survey in the Paediatric Day Care Unit, Barnsley DGH NHS Trust, South Yorkshire
}

\author{
A. M. Patel ${ }^{1}$
}

\begin{abstract}
Background In November 1998 the General Dental Council introduced guidelines for dental practitioners when referring a patient for general anaesthesia (GA). The practitioner is required to explain the risks associated with GA and the alternatives, give a detailed medical history and a clear justification for providing GA in the letter of referral.

Method A survey was administered on 202 parents or guardians, which aimed to investigate whether they felt that their dental practitioners had advised them of any risks of GA prior to referral. A record was also made if any reasons were given for the provision of GA in the letter of referral.

Results The majority of the parents or guardians (66\%) felt that they were not informed of any of the risks of GA and $25 \%$ felt that they were. From the letters of referral, 37\% contained a reason for $\mathrm{GA}$ and $63 \%$ did not give any reason or justification for $\mathrm{GA}$.

Conclusion There is evidence that referring practitioners do not adequately explain the risks of the anaesthetic to parents or guardians of children undergoing GA. There is also a lack of clear justification in the letters of referral for providing GA.

Practice implication It is essential that the alternatives and the risks of $\mathrm{GA}$ are discussed and if GA is still required, a clear justification should be contained in the letter of referral as part of informed consent. More importantly the referring practitioner should keep a contemporaneous record of this, preferably with a signature from the parent or guardian on agreement of referral.
\end{abstract}

The introduction of general anaesthesia (GA) in 1844 by $\mathrm{Dr}$ Horace Wells, revolutionized dental surgery by being the first reliable method of pain control. The subsequent advances in pharmacology and medicine have made the administration of GA much safer. However, safety is still dependent on suitable

${ }^{1 * S p e c i a l i s t ~ R e g i s t r a r ~ i n ~ O r t h o d o n t i c s, ~ O r t h o d o n t i c ~ D e p a r t m e n t, ~ B i r m i n g h a m ~ D e n t a l ~}$ Hospital, St. Chad's Queensway, Birmingham B4 6NN

${ }^{*}$ Correspondence to: Ashish Patel

Email:Ash_Patel_UK@Yahoo.com

\section{Refereed Paper}

Received 09.04.02; Accepted 15.05.03

doi:10.1038/sj.bdj.4811039

๑ British Dental Journal 2004; 196: 275-277 patient selection, the experience and training of staff, correct monitoring and an appropriate environment. The use of GA, despite these precautions, is associated with greater morbidity and mortality when compared with local anaesthetic or conscious sedation. In spite of such concerns, certain patients or procedures will, for the foreseeable future, require GA.

There have been numerous reports and reviews on the aspects of dental GA, but it was not until 1990 when the Poswillo Report ${ }^{1}$ was published that any universal guidelines had been produced. The report contained numerous recommendations, including the obligatory skills, training and qualifications of the clinical staff and auxiliaries, along with the necessary drugs and equipment required to provide GA in as safe a manner as possible. The recommendations also covered suitability and structure of a dental practice, which would lead to a further reduction of the risks.

The overall trend in the provision of GA was on the decline from the mid-1960s, however this was reversed in 1992 with the establishment of specialised practices offering GA on a referral basis. It was reported that, associated with the general decline of GA, the number of deaths had also decreased, although there was a small but significant rise in the late 1990s following an increase in general anaesthetic provision. ${ }^{2}$ Some of these deaths were widely publicised in the media with subsequent disciplinary actions by the General Dental Council (GDC), though a particular case in May 1998 caused the GDC to review its guidance in the provision of dental GA. ${ }^{3}$

Following this review in November 1998, the GDC implemented immediate revisions on the provision of GA for dental treatment. Among these changes was an increase in responsibility on the practitioner when referring their patient for a GA.

The referring practitioners now have to consider other means of pain and anxiety control and behavioural management. If these are not viable or successful, only then is GA considered an option. These practitioners also have the responsibility of discussing the risks involved with GA and alternative methods of pain and anxiety control with the patient, parent or guardian. Furthermore the GDC also requires the letter of referral to the specialist centre to contain a clear justification for providing GA. ${ }^{4}$ 
In line with these changes the British Dental Association (BDA) have produced an advice sheet for dental practitioners that provides "notes for handling GA cases as a basis of protocols to be agreed between all parties.' 5

Although the literature discusses the risks associated with GA, there are few reports that directly discuss the responsibilities of practitioners as outlined by the GDC guidelines.

\author{
Fig. 1 The patient survey form \\ The general anaesthetic (Gas) service at BDGH is currently being examined. As \\ part of this we would be grateful if you could answer the following five questions \\ in the shaded area of the questionnaire only. Please circle your answers. \\ 1. Were you present when your child was originally seen by your dentist? \\ Yes/No \\ 2. To your knowledge did the dentist have a look around all the teeth before \\ referring your child to hospital? \\ Yes/No/Don't know \\ 3. To your knowledge did the dentist take any $x$-rays of your child's teeth before \\ referring your child to hospital? \\ Yes/No/Don't know \\ 4. Has your dentist discussed with you any of the risks of General Anaesthesia \\ (Gas)? \\ Yes / No / Can't remember / Not present at the time \\ 5. To your knowledge has your child had any teeth removed under General \\ Anaesthesia (Gas) within the last year? \\ Yes / No / Can't remember \\ Thank you for your help. Please hand in this form to the doctor or nurse who will \\ be treating you today. \\ Addressograph \\ Date: \\ Referral Signature and Name: \\ Method of referral: Letter / PAF / Telephone / None / Other \\ Treatment requested: \\ Reason for request: \\ Treatment performed:
}

\section{AIM}

The aim of this study was to conduct a survey that investigated whether parents/guardians felt their dental practitioners, prior to referral had advised them of the risks of GA. The referral letters were analysed to determine whether a reason was given for the use of GA.

\section{METHOD}

An initial questionnaire was piloted, and following modification, five questions were devised for the parents or guardians to complete (Fig. 1) as part of a larger internal audit. Staff nurses distributed the questionnaires to the parents or guardians of all the children who attended the Day Case Unit at Barnsley District General Hospital. They were asked to complete the forms prior to the operation.

The age range of the patients was from 17 months to 15 years of age inclusive, all from the surrounding South Yorkshire region, and all referred for simple dental extractions under GA. The referring dental practitioners worked either in the general dental service or community dental service. As a prerequisite for day-case patients, all the patients were in either American Society of Anesthesiologists (ASA) classifications 1 or $2 .{ }^{6}$

A total of 202 questionnaires along with the accompanying referral letter and/or Paediatric Assessment Forms (PAFs) (Fig. 2)
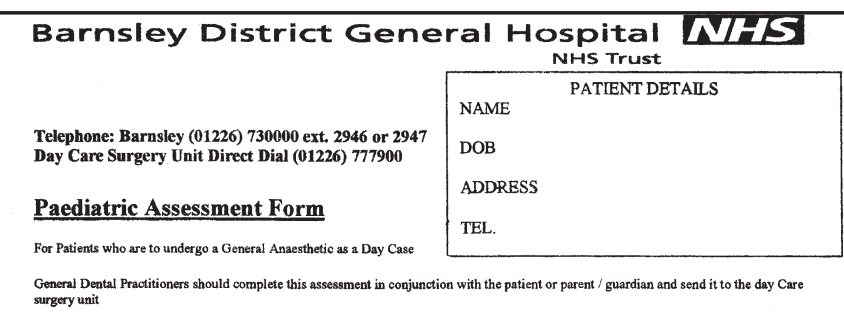

DATE AND TIME OF SURGERY AT THE HOSPITAL:

Extractions:

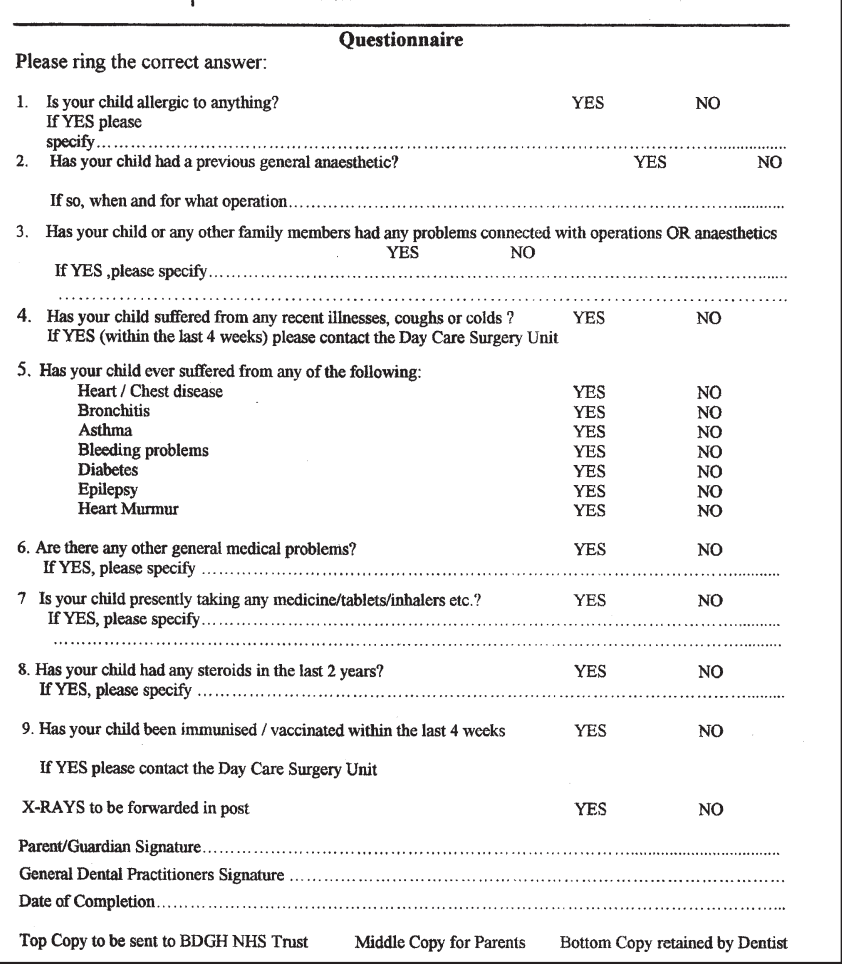

Fig. 2 The initial paediatric assessment form

were collected during a 5-month period, with 100\% compliance. These forms are printed proformas, designed by the dental department in conjunction with the day case unit of the hospital. They are distributed to the local practitioners on request and act as the letter of referral, medical questionnaire and pre and post-operative GA instructions for the patient. Any reasons given for referral on either the referral letter or PAF, by the practitioner, were duly recorded. These were collated by the author and analysed on a spreadsheet (Microsoft Excel 2000, Microsoft Corporation, USA).

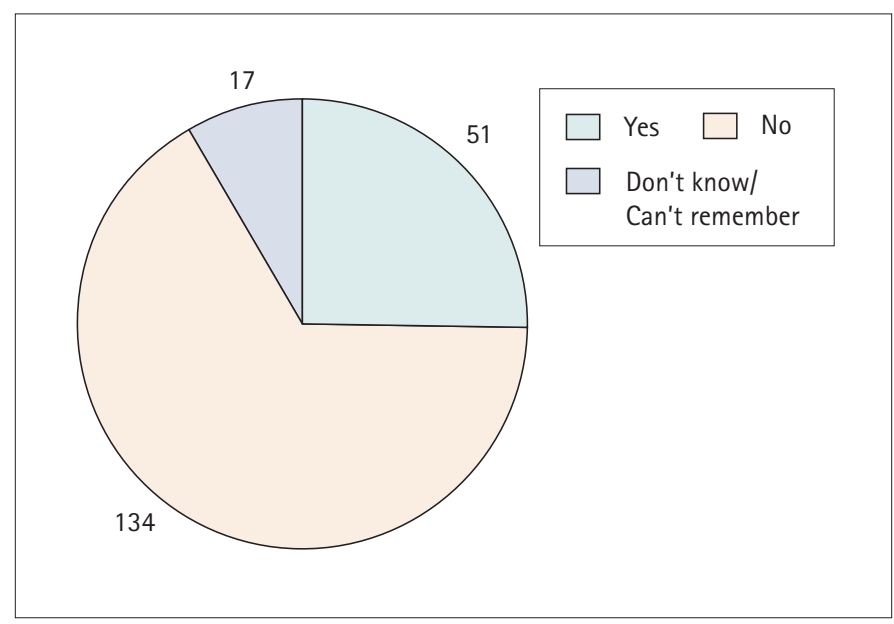

Fig. 3 The number of parents or guardians informed of the risks of GA by their referring practitioners 


\section{Number of referrals}

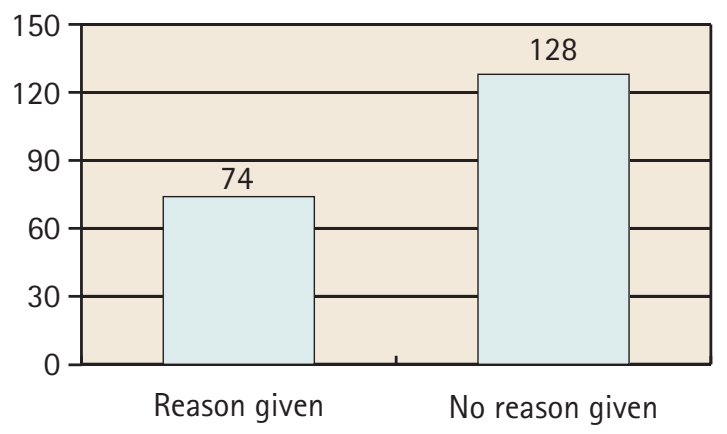

Fig. 4 Reason or justification given by the referring practitioner for GA

\section{RESULTS}

From the 202 questionnaires collected it was noted that 134 (66\%) parents or guardians felt that their practitioner did not adequately explain the risks of GA to them prior to referral. Fifty one (25\%) parents or guardians felt that the risks of GA were fully explained to them by their practitioner. It was found that $17(8 \%)$ of the parents or guardians were either not present at the visit of referral or could not remember the consultation (Fig. 3).

In the letters of referrals examined, 74 (37\%) gave a reason for requesting GA for the patient (Fig. 4), whereas 128 (63\%) referrals did not give any reason or justification for administering GA.

\section{DISCUSSION}

With the changes in the regulations for the provision of GA, which were brought into effect in 1998, the hospital services became the major providers of GA. In order to ease the path of referral and reduce delay for patients, many hospitals have developed a direct referral service where the patient sees the treating dentist and anaesthetist on the day of surgery. The success of this is however, reliant on the referring dental practitioner making an appropriate referral.

As seen in Maintaining Standards: Guidance to Dentists on Professional and Personal Conduct, ${ }^{4}$ paragraphs 4.18:

'the decision to refer a patient for treatment under GA should not be taken lightly. As part of this decision, a full medical history of the patient must be taken and agreement to refer obtained following a thorough and clear explanation of the risks involved and the alternative methods of pain control available. Clear justification for the use of GA, together with details of relevant medical and dental histories of the patient, must be contained in the referral. The referring dentist must retain a copy of this letter'

To provide informed consent, the patient has to be aware of the potential risks of GA, these include sore throat, nausea and vomiting, damage to teeth and awareness. The possibility of death is $1: 100,000$ as stated in the guidance sheet published by the BDA. ${ }^{5}$ Even though the risk of death is small, the consent is not informed if the patient, parent or guardian is not aware of this serious complication. ${ }^{7}$ As with any form of consent, it is important that these risks and options are discussed using appropriate language aimed at the patient's, parents' or guardians' level of understanding.
In order to ensure that the referring dentist, treating dentist and anaesthetist discuss the risks in a similar way it is prudent that there should be good communication between all three parties. Currently there is inadequate liaising and very few protocols in place for the proper referral of patients in many hospitals. More importantly it would be wise to keep a contemporaneous record of this discussion, which is important for medico-legal purposes. Ideally the letter of referral should also contain the parent or guardian's signature as consent for referral. The provision of a patient information leaflet can be a useful adjunct to verbal explanations.

The majority of practitioners who used the PAF alone, failed to provide any justification for the provision for GA (99\%). Such an omission however could be attributed to the PAF, where there is no specific section requesting justification. As a result of this study the day case unit will be making changes to the PAF and this will include a section that requests a reason for providing the patient with GA. Justification for providing GA would include, multiple extractions and or surgical procedures, a nervous and uncooperative child, allergy to local anaesthetic (LA), previously failed LA and certain mental or physical disabilities leading to poor compliance.

A drawback to this study is that it was based on the perceptions of parents or guardians. Parents or guardians may not remember what was said to them by their practitioner. Further work is necessary to determine how best to inform patients, parents or guardians in order to obtain informed consent.

However this study highlights that there is still some confusion surrounding GA. This confusion demonstrates the need for better information and publicity informing practitioners of their responsibilities with regards to GA referral. It is essential that the alternatives and the risks of GA are discussed and if GA is still required a clear justification should be contained in the letter of referral as part of informed consent. More importantly the referring practitioner should keep a contemporaneous record of this, preferably with a signature from the parent or guardian on agreement of referral.

\section{CONCLUSION}

There is evidence that referring practitioners, of the study population, do not adequately explain the risks of the anaesthetic to parents or guardians of children undergoing GA. There is also a lack of clear justification in the letters of referral for providing GA.

The author would like to thank Mr J. D. Price, Consultant Oral Surgeon, the staff and patients on day case surgery, Barnsley District General Hospital. Also Professor D. Walmsley, Birmingham Dental School for proof reading the manuscript.

1. Poswillo D. General Anaesthesia, Sedation and Resuscitation in Dentistry. Report of an Expert Working Party for the Standing Dental Advisory Committee. Department of Health, 1990.

2. Chief Medical Officer/Chief Dental Officer (England) Report. A Conscious Decision: A review of the use of general anaesthesia and conscious sedation in primary care. Department of Health, 2000. www.doh.gov.uk/dental/conscious.htm

3. Minutes from Professional Conduct Committee. Case of Schonberg, Item 6, May 1998. Chaired by Steward M., General Dental Council Meeting. November, 1998.

4. General Dental Council. Maintaining Standards: Guidance to Dentists on Professional and Personal Conduct. London: General Dental Council, November 1998 and May 1999.

5. Notes for handling general anaesthesia cases. British Dental Association Publication, 1999.

6. American Society of Anesthesiologists: New classification of physical status, Anesthesiol 1963, 24: 111.

7. Sidaway vs Board of Governors of Bethlem Royal and the Maudsley Hospital case. Ethics in dentistry, BDA advice publication B1, June 2000. 